\title{
Erratum: Competitive Analysis for Multi-Objective Online Algorithms
}

\author{
Morten Tiedemann (凶), Jonas Ide, and Anita Schöbel \\ DFG RTG 1703, Institute for Numerical and Applied Mathematics, \\ University of Göttingen, Lotzestr. 16-18, D-37083 Göttingen, Germany \\ \{m.tiedemann, j.ide, schoebel\}@math.uni-goettingen.de
}

DOI 10.1007/978-3-319-15612-5_31

We identify an error in the analysis of the online algorithm RPP-MULT for the bi-objective time series search problem presented in [1]. The strong competitive ratio with respect to $f_{2}(c)=\frac{1}{k} \sum_{i=1}^{k} c_{i}$ of RPP-MULT stated in [1, Theorem 3] does not hold due to an error in the proof. In the following, we point out this error. For details about the problem and the terminology, we refer to the original paper.

In the course of the proof of [1, Theorem 3], the expression

$$
\max _{x \in \mathcal{I}_{1}}\left\{\frac{M_{1}}{x}+\frac{M_{2} x}{z^{\star}}\right\}
$$

is considered (see Equation (9)). Here, the maximum is falsely determined as $x^{*}=\sqrt{\left(M_{1} z^{\star}\right) / M_{2}}$. In fact, $x^{*} \notin \mathcal{I}_{1}$, and, thus, Equation (9) does not hold. The same mistake has been made in Equation (11), i.e., the chosen maximum is not a point in $\mathcal{I}_{2}$.

Note that the proof cannot be modified such that the stated competitive ratio is proven. However, in [2], a best possible algorithm for the bi-objective time series search problem is presented, as outlined below.

Hasegawa and Itoh define the online algorithm Balanced Price Policy $\mathrm{BPP}_{k}$ for the $k$-objective time series search problem with respect to an arbitrary monotone continuous function $f: \mathbb{R}^{k} \rightarrow \mathbb{R}$, see Algorithm 1 .

$$
\begin{aligned}
& \text { for } t=1,2, \ldots, T \text { do } \\
& \text { Accept } \boldsymbol{p}_{t}=\left(p_{t}^{1}, \ldots, p_{t}^{k}\right)^{\top} \text { if } f\left(\frac{M_{1}}{p_{t}^{1}}, \ldots, \frac{M_{k}}{p_{t}^{k}}\right) \leq f\left(\frac{p_{t}^{1}}{m_{1}}, \ldots, \frac{p_{t}^{k}}{m_{k}}\right) \text {. } \\
& \text { end }
\end{aligned}
$$

Algorithm 1: Balanced Price Policy $\mathrm{BPP}_{k}$ 
Let $z_{k}^{f}=\sup _{\left(x_{1}, \ldots, x_{k}\right) \in \mathcal{S}_{f}^{k}} f\left(\frac{M_{1}}{x_{1}}, \ldots, \frac{M_{k}}{x_{k}}\right)$, where

$$
\mathcal{S}_{f}^{k}=\left\{\left(x_{1}, \ldots, x_{k}\right) \in I_{1} \times \cdots \times I_{k}: f\left(\frac{M_{1}}{x_{1}}, \ldots, \frac{M_{k}}{x_{k}}\right)=f\left(\frac{x_{1}}{m_{1}}, \ldots, \frac{x_{k}}{m_{k}}\right)\right\}
$$

with $I_{i}=\left[m_{i}, M_{i}\right]$ for $i=1, \ldots, k$.

Hasegawa and Itoh prove that, for any integer $k \geq 1$ and any monotone continuous function $f: \mathbb{R}^{k} \rightarrow \mathbb{R}$, the competitive ratio of $\mathrm{BPP}_{k}$ with respect to $f$ is given by $z_{f}^{k}$ and this is the best possible competitive ratio, see [2, Section 4.1].

By means of this result, the following theorem gives the best possible competitive ratio with respect to $f_{2}$ and $k=2$ :

Theorem 1 (Hasegawa and Itoh, [2, Theorem 6.1]). With respect to the function $f_{2}$ for $k=2$, the following holds:

$$
z_{f_{2}}^{2}=\frac{1}{2}\left[\sqrt{\left(\frac{1}{2}\left(\frac{M_{2}}{m_{2}}-1\right)\right)^{2}+\frac{M_{1}}{m_{1}}}+\frac{1}{2}\left(\frac{M_{2}}{m_{2}}+1\right)\right] .
$$

\section{References}

1. Tiedemann, M. and Ide, J. and Schöbel, A.: Competitive Analysis for MultiObjective Online Algorithms. In: Proceedings of the 9th Workshop on Algorithms and Computation (WALCOM). LNCS, vol. 8973, pp. 210-221 (2015)

2. Hasegawa, S. and Itoh, T.: Optimal Online Algorithms for the Multi-Objective Time Series Search Problem. arXiv:1506.04474v4 [cs.DS] (2015) 